\title{
MISCELLANEOUS
}

\section{THE CONCEPT OF ASYMPTOTIC EFFICIENCY}

By D. BASU

Indian Statistical Institute, Calcutta

\section{Summary}

Partly of an expository nature this note brings out the fact that an estimator, though asymptotically much less efficient (in the classical sense) than another, may yet have much greater probability concentration (as defined in this article) than the latter.

\section{Definitions}

Let $\left\{X_{i}\right\}, i=1,2, \ldots$ be an infinite sequence of independent and identically distributed random variables whose common distribution function $F$ is known to belong to a family $\Omega$ of one dimensional distribution finctions. Let $\mu=\mu(F)$ be a real valued functional defined on $\Omega$. By an estimator $T=\left\{t_{n}\right\}$ of $\mu$ we mean a sequence of real valued measurable functions of $\left\{X_{i}\right\}$, where $t_{n}$ is a function of $X_{1}, X_{2}, \ldots, X_{n}$ only $(n=1,2, \ldots)$. The estimator $T$ is said to be an asymptotically normal estimator of $\mu$ if there exists a sequence $\left\{\sigma_{n}\left(F^{\prime}\right)\right\}$ of positive numbers such that as $n \rightarrow \infty$

$$
\left\{t_{n}-\mu(F) / \sigma_{n}(F)\right\} \Longrightarrow N(0,1) \quad \text { for all } F \varepsilon \Omega
$$

where $\Longrightarrow$ stands for convergence in law and $N(0,1)$ for the standard normal variable. The sequence $\left\{\sigma_{n}(F)\right\}$ is called the asymptotic standard deviation of $T$. A necessary and sufficient condition in order that $\left\{\sigma_{n}(F)\right\}$ and $\left\{\sigma_{n}^{\prime}(F)\right\}$ may both be called the asymptotic standard deviation of $T$ is

$$
\lim _{n \rightarrow \infty}\left\{\sigma_{n}(F) / \sigma_{n}^{\prime}(F)\right\} \equiv 1 \quad \text { for all } F \varepsilon \Omega
$$

A necessary and sufficient condition in order that the asymptotically normal estimator $T$ is also consistent is

$$
\lim _{n \rightarrow \infty} \sigma_{n}(F) \equiv 0 \quad \text { for all } F \varepsilon \Omega
$$

Let $\mathcal{Z}$ be the family of all consistent asymptotically normal estimators of $\mu$. We consider only the space $\mathcal{Z}$. 


\section{THE PARTIAL ORDER OF EFFICIENCY}

Two elements $T$ and $T^{\prime}$ of $\mathcal{Z}$ are said to be equally efficient (or equivalent) if they have the same asymptotic s.d.s, i.e. if

$$
\lim _{n \rightarrow \infty}\left\{\sigma_{n}(F) / \sigma_{n}^{\prime}\left(F^{\prime}\right)\right\} \equiv 1 \quad \text { for all } F \varepsilon \Omega
$$

where $\left\{\sigma_{n}(F)\right\}$ and $\left\{\sigma_{n}^{\prime}(F)\right\}$ are the corresponding asymptotic s. d.'s.

It is easily verified that the above equivalence relation is reflexive, symmetric, and transitive.

$$
\text { If } \quad \limsup _{n \rightarrow \infty}\left\{\sigma_{n}(F) / \sigma_{n}^{\prime}(F)\right\} \leqslant 1 \quad \text { for all } F \varepsilon \Omega
$$

and

$$
\liminf _{n \rightarrow \infty}\left\{\sigma_{n}(F) / \sigma_{n}^{\prime}\left(F^{\prime}\right)\right\}<1 \quad \text { for some } F \varepsilon \Omega
$$

then we say that $T$ is more efficient than $T^{\prime}$ and write $T \supset T^{\prime}$. It is easily seen that the relation $\supset$ induces a partial order on $\mathcal{Z}$.

It is known that there do not exist a maximal element in $\mathcal{Z}$ with respect to the partial order $\supset$, i.e. there do not exist any element $T \varepsilon \mathcal{I}$ which is either equivalent to or more efficient than any alternative $T^{\prime \prime} \varepsilon \mathcal{I}$. As a matter of fact it has been demonstrated (LeCam, 1953) how given any $T \varepsilon \mathcal{I}$ we can always find a $T^{\prime} \varepsilon \mathcal{Z}$ such that $T^{\prime} \supset T$.

\section{The PaRtial ORDer OF CONCENTRATion}

The estimator $T=\left\{t_{n}\right\}$ of $\mu$ is consistent if for all $\epsilon>0$ and $F \varepsilon \Omega$

$$
p_{n}(\epsilon, F)=P\left\{\left|t_{n}-\mu\right|>\epsilon \mid F\right\} \rightarrow 0 \text { as } n \rightarrow \infty .
$$

If we work with the simple loss function that is zero or one according as the error in the estimate is $\leqslant \epsilon$ or $>\epsilon$ then $p_{n}(\epsilon, F)$ is the risk (or expected loss) when the estimator is used with observations on $X_{1}, X_{2}, \ldots, X_{n}$ only.

The rapidity with which $p_{n}\left(\epsilon, F^{\prime}\right) \rightarrow 0$ may be considered to be a measure of the asymptotic accuracy or concentration of $T$. This motivates the following definition of a partial order on $\mathcal{I}$ (and as a matter of fact on the wider family of all consistent estimators of $\mu$ ).

Definition: The estimator $T$ with the associated sequences of risk functions $p_{n}\left(\epsilon, F^{\prime}\right)$ is said to have greater concentration than $T^{\prime \prime}$ with the associated sequences $p_{n}^{\prime}(\epsilon, F)$ if, for all $\epsilon>0$ and $F \varepsilon \Omega$,

$$
\limsup _{n \rightarrow \infty}\left\{p_{n}(\epsilon, F) / p_{n}^{\prime}(\epsilon, F)\right\} \leqslant 1
$$

with the limit inferior being $<1$ for some $\epsilon>0$ and some $F \varepsilon \Omega$. We then write $T>T^{\prime \prime}$. 


\section{THE CONCEPT OF ASYMPTOTIC EFFICIENCY}

Intuitively it may seem reasonable to expect that $T \supset T^{\prime}$ implies $T>T^{\prime}$. That this is not so is demonstrated in the next section. An example is given where

$$
\lim _{n \rightarrow \infty} \frac{\sigma_{n}(F)}{\sigma_{n}^{\prime}(F)} \equiv 0 \quad \text { for all } \vec{F} \varepsilon \Omega
$$

whereas

$$
\lim _{n \rightarrow \infty} \frac{p_{n}(\epsilon, F)}{p_{n}^{\prime}(\epsilon, \bar{F})} \equiv \infty \quad \text { for all } \epsilon>0 \text { and } F \varepsilon \Omega
$$

\section{AN EXAMPLE}

Let each of the $X_{i}$ 's be $N(\mu, 1)$, the problem being to estimate $\mu$.

Let

$$
\hat{X}_{n}=\sum_{1}^{n} X_{i} / n \text { and } S_{n}=\sum_{1}^{n}\left(X_{i}-\bar{X}_{n}\right)^{2}
$$

Then $\vec{X}_{n}$ and $S_{n}$ are mutually independent random variables and the distribution of $S_{n}$ is independent of $\mu$. Let $a_{n}$ be the upper $100 / n \%$ point of $S_{n}$ and let

$$
H_{n}=\left\{\begin{array}{lll}
0 & \text { if } & S_{n} \leqslant a_{n} \\
1 & \text { if } & S_{n}>a_{n} .
\end{array}\right.
$$

Now let

$$
T=\left\{t_{n}\right\}
$$

where

$$
t_{n}=\left(\mathrm{l}-H_{n}\right) \overline{\mathrm{X}}_{n}+n H_{n}
$$

anḍ

$$
T^{\prime}=\left\{t_{n}^{\prime}\right\}
$$

where

$$
t_{n}^{\prime}=\overline{\mathbf{X}}_{[\sqrt{n}]} .
$$

(By $[x]$ we mean the largest integer not exceeding $x$.)

Since

$$
P\left(H_{n}=0\right)=1-\frac{1}{n} \rightarrow 1
$$

it follows (vide Cramér, p. 254) that $\sqrt{n}\left(t_{n}-\mu\right)=\sqrt{n}\left(\bar{X}_{n}-\mu\right)+\sqrt{n} H_{n}\left(n-\vec{X}_{n}\right)$

$$
\Longrightarrow N(0,1)
$$

when $\mu$ is the true mean.

Hence, $T \varepsilon \mathcal{I}$ with asymptotic s.d. $=\left\{n^{-1 / 2}\right\}$. Also $T^{\prime} \in \mathcal{I}$ with asymptotic s.d. $=\left\{n^{-1 / 4}\right\}$. 
Therefore (4.1) is satisfied. Again, since $\bar{X}_{n}$ is independent of $H_{n}$ it follows that, for every $n>\mu+\varepsilon$,

$$
\begin{aligned}
P\left(\left|t_{n}-\mu\right|>\epsilon \mid \mu\right) & =P\left(H_{n}=0\right) P\left(\left|\bar{X}_{n}-\mu\right|>\epsilon \mid \mu\right)+P\left(H_{n}=1\right) \\
& =\frac{1}{n}+o\left(\frac{1}{n}\right)
\end{aligned}
$$

because

$$
P\left(\left|\bar{X}_{n}-\mu\right|>\epsilon \mid \mu\right)=o\left(\frac{1}{n}\right), \text { as may be easily verified. }
$$

Whereas

$$
P\left(\left|t_{n}^{\prime}-\mu\right|>\epsilon \mid \mu\right)=o\left(\frac{1}{n}\right)
$$

Therefore (4.2) also is satisfied.

It may be noted that in the example given the s.d. of $t_{n}$ is not asymptotically equal to the asymptotic s.d. of $T$. But this can be easily arranged to be true by, say, taking $a_{n}$ for the upper $100 / n^{4} \%$ point of $S_{n}$.

\section{REFERENCES}

CRAmḱr, H. (1946) : Methematical Methods of Statistics, Princeton University Press.

LECAM, L. (1953): On Some Asymptotic Properties of Maximum Likelihood Estimates and Related Bayes Estimates, University of California Press.

Paper received : January, 1955. 